\title{
UNSTEADY MOTION OF VISCOUS ELECTRICALLY CONDUCTIVE FLUID ROTATING IN HALF-SPACE BOUNDED BY A WALL IN THE PRESENCE OF MEDIUM INJECTION (SUCTION)
}

\author{
A.A. Gurchenkov ${ }^{1,2}$ \\ challenge2005@mail.ru \\ ${ }^{1}$ Bauman Moscow State Technical University, Moscow, Russian Federation \\ ${ }^{2}$ Federal Research Centre for Computer Science and Control, \\ Russian Academy of Sciences, Moscow, Russian Federation
}

\begin{abstract}
The study is devoted to studying motion of a viscous electrically conductive incompressible fluid, which initially rotates as a solid body with constant angular velocity together with a porous wall bounding it under the influence of suddenly appearing longitudinal oscillations of the wall. The wall forms an arbitrary angle with the axis of rotation. Unsteady flow is induced by longitudinal wall oscillations, injection (suction) of the medium directed perpendicular to the porous plate surface and by suddenly activated constant magnetic field directed on the normal to the plate. Solutions were constructed for velocity fields and fluid pressure. Induced magnetic field in the flow of electrically conductive fluid was determined. A number of particular cases of the wall motion were considered. Based on the results obtained, separate structures of the boundary layers adjacent to the wall were examined
\end{abstract}

\section{Keywords}

Magnetic hydrodynamic equations, electrically conductive fluid, normal oscillations, boundary layers

Accepted 12.07.2019

(c) Author(s), 2020

Introduction. This study summarizes the previous results [1-3]. It is demonstrated that in the absence of the medium injection (suction) and in the absence of a magnetic field the solution coincides with the results of paper [3], and in the absence of rotation, magnetic field and medium transverse flow the given solution is becoming a solution to the problem of unsteady motion of viscous fluid bounded by a moving flat wall [1].

In the recent years, interest aroused to electromagnetic pumps and measuring instruments based on magnetic hydrodynamic effects in connection with development in technologies of nuclear reactors with metal heat carriers [4-9].

Studying the effect of spontaneous generation of a magnetic field is important from the practical point of view. Cooling of the nuclear reactors is provided by using the liquid sodium. If the reactor size is increasing, volumes of 
A.A. Gurchenkov

liquid metal are also rising, and the metal is moving at a high velocity, and its conductivity is very high. As a result, a magnetic field could spontaneously arise, which would alter the process of heat and mass transfer in the reactor. Such a possibility should be foreseen and prevented [10-14].

Research in the magnetic hydrodynamics area has also other practical applications, especially important in metallurgy. The fact is that an electromagnetic field actively affects the liquid metal. Changing the magnetic field magnitude and its configuration, as well as the electric current that passes through the liquid metal, could lead to ensuring control over its flow [15-19].

Analytical solutions of magnetic hydrodynamic equations. The present work studies unsteady flow of a viscous electrically conductive incompressible fluid in a half space bounded by an infinite flat wall, to which normal the medium injection (suction) is performed. The fluid and the plate are being in a state of rotation as a solid body with constant angular velocity constituting the $\beta$ angle to the plate, $0<\beta<\pi / 2$. Unsteady flow is induced by longitudinal wall oscillations and medium injection (suction) produced at the $\vec{a}(t)$ velocity perpendicular to the porous plate surface and incidentally introduced magnetic field with the $\vec{B}_{0}=$ const induction directed in the normal to the plate. It turned out that in this case, an exact solution could be found to the three-dimensional nonstationary equations of magnetic hydrodynamics. Schematically, the problem statement is presented in Fig. 1.

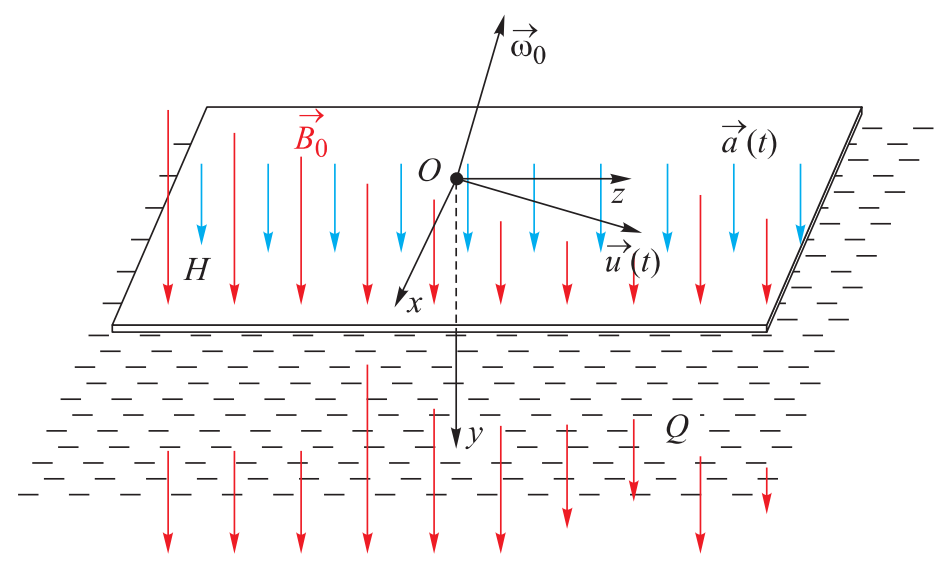

Fig. 1. Schematic problem statement under consideration

Let us consider incompressible fluid having $\rho$ density, $v$ kinematic viscosity, $\mu$ magnetic permeability and $\sigma$ electrical conductivity, which fills the $Q$ half-space bounded by the $H$ porous wall. The fluid and the wall are rotating as an integral unit with the $\vec{\omega}_{0}=$ const angular velocity around the direction not perpendicular to the plate. 
Nonstationary flow is induced by incidentally starting longitudinal oscillations of the wall, which begins to move with the $\vec{u}(t)$ speed. Medium injection (suction) is performed through the porous plate surface at the $\vec{a}(t)$ velocity along the normal to the $H$ wall. In addition, constant magnetic field with the $B_{0}$ induction is applied along the normal to the plate surface.

Let us connect the $O x y z$ Cartesian coordinate system with the $H$ plate in such a way that the $O x z$ plane coincides with the $\mathrm{H}$ plane, and the $O y$ axis is directed inside the fluid. Let $\vec{e}_{x}, \vec{e}_{y}$ and $\vec{e}_{z}$ be the unit vectors of the Cartesian coordinate system. Fluid motion in the Oxyz system rotating at the $\vec{\omega}_{0}$ angular velocity in the magnetic hydrodynamics approximation (infinitely conductive fluid) is described by magnetic hydrodynamics equations:

$$
\begin{aligned}
& (\vec{v} \nabla) \vec{v}+\frac{\partial \vec{v}}{\partial t}+2 \vec{\omega}_{0} \times \vec{v}=-\nabla P+v \Delta \vec{v}+\frac{1}{\mu \rho} \operatorname{rot} \vec{B} \times \vec{B} ; \\
& \operatorname{div} \vec{v}=0 ; \vec{r} \in Q ; \\
& \frac{\partial \vec{B}}{\partial t}=\operatorname{rot} \vec{v} \times \vec{B} ; \\
& \operatorname{div} \vec{B}=0 ; \vec{r} \in Q ;
\end{aligned}
$$

as well as by boundary and initial conditions, which in the usual notations have the following form:

$$
\begin{aligned}
& \vec{v}=\left(\vec{u}(t), \vec{a}(t) \vec{e}_{y}\right) \text { at } \vec{r} \in H, t \geq 0 ; \\
& \vec{B}=B_{0} \vec{e}_{y} \text { at } \vec{r} \in H ; \\
& |\vec{v}| \rightarrow 0 \text { at }|\vec{r}| \rightarrow \infty, t>0 ; \\
& |\vec{B}|=B_{0} \text { at }|\vec{r}| \rightarrow \infty, t>0
\end{aligned}
$$

and

$$
\begin{aligned}
& \vec{v}(\vec{r}, 0)=0 \text { at } t=0 ; \\
& \vec{B}(\vec{r}, 0)=0 \text { at } t=0 .
\end{aligned}
$$

Here $\vec{r}$ is the radius vector relative to the $O$ pole; $\vec{v}$ is the fluid velocity; $P$ is the pressure; $\rho$ is the density; $\nu$ is the fluid kinematic viscosity; $\mu$ is the magnetic permeability and $\vec{B}$ is the magnetic induction.

In the system of equations (1), the $U$ centrifugal forces and gravitational potential are included in the $P$ pressure.

Solution to the system of equations (1) could be found in the following form:

$$
\begin{aligned}
& \vec{v}=\left\{v_{x}(y, t), a(t), v_{z}(y, t)\right\} ; \\
& P=2 \omega_{0 z} a(t) x-2 \omega_{0 x} a(t) z-\frac{\partial a(t)}{\partial t} y+q(y, t),
\end{aligned}
$$

where $q(y, t)$ is the unknown pressure function, $\omega_{0 x}=\vec{\omega}_{0} \vec{e}_{x}$ and $\omega_{0 z}=\vec{\omega}_{0} \vec{e}_{z}$. 
To determine the $\vec{v}$ velocity and the $\vec{B}$ induction field, the following system of equations is obtained:

$$
\begin{aligned}
& \frac{\partial v_{x}}{\partial t}+2 \omega_{0 y} v_{z}=v \frac{\partial^{2} v_{x}}{\partial y^{2}}-a(t) \frac{\partial v_{x}}{\partial y}+\frac{1}{\mu \rho} B_{0} \frac{\partial B_{x}}{\partial y} \\
& \frac{\partial v_{z}}{\partial t}-2 \omega_{0 y} v_{x}=v \frac{\partial^{2} v_{z}}{\partial y^{2}}-a(t) \frac{\partial v_{z}}{\partial y}+\frac{1}{\mu \rho} B_{0} \frac{\partial B_{z}}{\partial y} \\
& \frac{\partial q}{\partial y}=2 \vec{v}\left(\vec{\omega}_{0} \times \vec{e}_{y}\right)-\frac{1}{\mu \rho}\left(B_{x} \frac{\partial B_{x}}{\partial y}+B_{z} \frac{\partial B_{z}}{\partial y}\right) ; \\
& \frac{\partial B_{x}}{\partial t}=B_{0} \frac{\partial v_{x}}{\partial y} \\
& \frac{\partial B_{z}}{\partial t}=B_{0} \frac{\partial v_{z}}{\partial y}
\end{aligned}
$$

boundary and initial conditions:

$$
\begin{gathered}
\vec{v}(y, t)=\left(\vec{u}(t), a(t) \vec{e}_{y}\right) \text { at } y=0 \text { and } t>0 ; \\
\vec{B}(y, t)=B_{0} \vec{e}_{y} \text { at } y=0 \text { and } t>0 ; \\
|\vec{v}(y, t)| \rightarrow 0 \text { at } y \rightarrow \infty \text { and } t>0 ; \\
B_{x}, B_{z} \rightarrow 0 \text { at } y \rightarrow \infty \text { and } t>0 ; \\
\vec{v}(y, 0)=0 \text { at } t=0 \text { and } y>0 .
\end{gathered}
$$

Let us consider a case, where all the values in (5) and (6) depend on time through the $e^{\lambda t}$ factor, and $\lambda$ is the complex number (quasi-harmonic mode). In addition, the injection velocity is set as $a(t)=a=$ const. It should be noted that $a>0$ corresponds to the medium injection and $a<0-$ to the medium suction.

In this case, the system of equations (5) and the boundary conditions (6) could be written in the following form:

$$
\begin{aligned}
& \lambda v_{x}+2 \Omega v_{z}=v \frac{\partial^{2}}{\partial y^{2}} v_{x}-a \frac{\partial}{\partial y} v_{x}+\frac{B_{0}}{\mu \rho} \frac{\partial}{\partial y} B_{x} \\
& \lambda v_{z}-2 \Omega v_{x}=v \frac{\partial^{2}}{\partial y^{2}} v_{z}-a \frac{\partial}{\partial y} v_{z}+\frac{B_{0}}{\mu \rho} \frac{\partial}{\partial y} B_{z} \\
& \lambda B_{x}=B_{0} \frac{\partial}{\partial y} v_{x} ; \lambda B_{z}=B_{0} \frac{\partial}{\partial y} B_{z} .
\end{aligned}
$$

Here $\Omega=\vec{\omega}_{0} \vec{e}_{y}$. 
Excluding the $\left(B_{x}, B_{z}\right)$ magnetic field from equations (7) using the $B_{x}=\frac{B_{0}}{\lambda} \frac{\partial}{\partial y} v_{x}$ and $B_{z}=\frac{B_{0}}{\lambda} \frac{\partial}{\partial y} v_{z}$ formulas, three systems of equations are obtained for determining the $\vec{v}$ velocity field, the $\vec{B}$ magnetic induction and the $q(y, t)$ pressure field:

$$
\begin{gathered}
\lambda v_{x}+2 \Omega v_{z}=\left(v+\frac{B_{0}^{2}}{\mu \rho \lambda}\right) \frac{\partial^{2}}{\partial y^{2}} v_{x}-a \frac{\partial}{\partial y} v_{x} ; \\
\lambda v_{z}-2 \Omega v_{x}=\left(v+\frac{B_{0}^{2}}{\mu \rho \lambda}\right) \frac{\partial^{2} v_{z}}{\partial y^{2}}-a \frac{\partial}{\partial y} v_{z} ; \\
v_{x}(0)=u_{x} ; v_{z}(0)=u_{z} \text { at } y=0 ; v_{x}, v_{z} \rightarrow 0 \text { at } y \rightarrow \infty ; \\
B_{x}=\frac{B_{0}}{\lambda} \frac{\partial}{\partial y} v_{x} ; \\
B_{z}=\frac{B_{0}}{\lambda} \frac{\partial}{\partial y} v_{z}, B_{x}, B_{z} \rightarrow 0 \text { at } y \rightarrow \infty ; \\
\frac{\partial q}{\partial y}=2 \vec{v}\left(\vec{\omega}_{0} \times \vec{e}_{y}\right)-\frac{1}{2 \mu \rho} \frac{\partial}{\partial y}\left(B_{x}^{2}+B_{z}^{2}\right) ; \\
q(y, t) \rightarrow 0 \text { at } y \rightarrow \infty .
\end{gathered}
$$

System of equations (8) is used to determine the velocity field, system (9) is used to determine the $\vec{B}$ induction, and equation (10) is used to determine the pressure field from the indicated velocity field and induction.

Let us introduce a complex structure:

$$
\hat{v}=v_{x}+i v_{z} ; \hat{B}=B_{x}+i B_{z} ; \hat{u}=u_{x}+i u_{z}
$$

Then the system of equations (8) and (9) will take the following form:

$$
\begin{aligned}
& \lambda \hat{v}-i \cdot 2 \Omega \hat{v}=\left(v+\frac{B_{0}^{2}}{\mu \rho \lambda}\right) \frac{\partial^{2}}{\partial y^{2}} \hat{v}-a \frac{\partial}{\partial y} \hat{v} ; \\
& \hat{B}=\frac{B_{0}}{\lambda} \frac{\partial \hat{v}}{\partial y} .
\end{aligned}
$$

To determine the $\vec{v}$ velocity field, let us use the ordinary differential equation of the second order:

$$
\begin{aligned}
& \left(v+\frac{B_{0}^{2}}{\mu \rho \lambda}\right) \frac{\partial^{2}}{\partial y^{2}} \hat{v}-a \frac{\partial \hat{v}}{\partial y}-(\lambda-i \cdot 2 \Omega) \hat{v}=0 ; \\
& \hat{v} \rightarrow 0 \text { at } y \rightarrow \infty ; \\
& \hat{v}(0)=\hat{u}(0) .
\end{aligned}
$$


Characteristic equation (12)

$$
\left(v+\frac{B_{0}^{2}}{\mu \rho \lambda}\right) \chi^{2}-a \chi-(\lambda-i \cdot 2 \Omega)=0 .
$$

Equation roots

$$
\chi_{1,2}=\frac{a}{2\left(v+\frac{B_{0}^{2}}{\mu \rho \lambda}\right)} \pm \sqrt{\frac{a^{2}}{4\left(v+\frac{B_{0}^{2}}{\mu \rho \lambda}\right)^{2}}+\frac{\lambda-i \cdot 2 \Omega}{v+\frac{B_{0}^{2}}{\mu \rho \lambda}}} ; \quad \operatorname{Re} \chi_{1,2} \leq 0 .
$$

If $\lambda \neq \pm i \cdot 2 \Omega$, solution (12), taking into consideration the boundary conditions, will take the following form:

$$
\hat{v}(y)=\hat{u}(0) \frac{E_{1}+E_{2}}{2}+i \hat{u}(0) \times \vec{e}_{y} \frac{E_{1}-E_{2}}{2},
$$

where $E_{j}=\exp \left(\chi_{j} y\right), j=1,2$, velocity field is

$$
\hat{v}(y, t)=e^{\lambda t}\left[\hat{u}(0) \frac{E_{1}+E_{2}}{2}+i \hat{u}(0) \times \vec{e}_{y} \frac{E_{1}-E_{2}}{2}\right],
$$

fluid pressure

$$
\begin{gathered}
q(y, t)=\frac{\left(\vec{\omega}_{0} \times \vec{e}_{y}\right)}{2}\left[\hat{u}(0)\left(\frac{1}{\chi_{1}}+\frac{1}{\chi_{2}}\right)+\hat{u}(0) \times \vec{e}_{y}\left(\frac{1}{\chi_{1}}-\frac{1}{\chi_{2}}\right)\right] e^{\lambda t}- \\
-\frac{1}{2} \frac{1}{\mu \rho} \hat{B}(y, t)^{2}
\end{gathered}
$$

and induction vector is

$$
\hat{B}(y, t)=e^{\lambda t}\left[\hat{u}(0) \frac{\chi_{1} E_{1}+\chi_{2} E_{2}}{2}+i \hat{u}(0) \times \vec{e}_{y} \frac{\chi_{1} E_{1}-\chi_{2} E_{2}}{2}\right] \frac{B_{0}}{\lambda} .
$$

Let us consider the resonance case at

$$
\vec{v}(y, t)=e^{\lambda t}\left[\vec{u}(0) \frac{E_{1}+1}{2}+i \vec{u}(0) \times \vec{e}_{y} \frac{E_{1}-1}{2}\right] .
$$

Here

$$
E_{1}=\exp \left(\frac{a y}{\nu+B_{0}^{2} /(\mu \rho \lambda)}\right) ; E_{2}=1
$$

at $\lambda=-i \cdot 2 \Omega$ 


$$
\vec{v}(y, t)=e^{\lambda t}\left[\vec{u}(0) \frac{1+E_{2}}{2}+i \vec{u}(0) \times \vec{e}_{y} \frac{1-E_{2}}{2}\right] ;
$$

here

$$
E_{1}=1 ; E_{2}=\exp \left(\frac{a y}{v+B_{0}^{2} /(\mu \rho \lambda)}\right) .
$$

In both cases, at $a<0$ and $y \rightarrow \infty$, the velocity field

$$
\vec{v}(y, t)=\frac{1}{2} e^{\lambda t}\left[\vec{u}(0) \mp i \vec{u}(0) \times \vec{e}_{y}\right]
$$

is oscillatory in nature and is not tending to zero remaining a limitation.

Solution in this resonance case satisfies the boundary conditions on the $H$ plate, but does not satisfy the conditions for infinity, i.e., a so-called hydrodynamic paradox is taking place.

Boundary layers structure. Let us study expression (13) in more detail. Let the plate move at the velocity of $\vec{u}(t)=\vec{u}(0) e^{\lambda t}, \lambda=-\alpha+i \omega$.

A case will be considered, when any injection (suction) of fluid through the plate surface is missing. Then the velocity field of viscous electrically conductive liquid will have the following form:

$$
\vec{v}(y, t)=e^{(-\alpha+i \omega) t}\left[\vec{u}(0) \frac{E_{1}+E_{2}}{2}+i \vec{u}(0) \times \vec{e}_{y} \frac{E_{1}-E_{2}}{2}\right],
$$

where $E_{1}=e^{\mu_{1} y} ; \quad E_{2}=e^{\mu_{2} y} ; \quad \mu_{1,2}=\sqrt{\frac{\lambda \mp i \cdot 2 \Omega}{\nu+B_{0}^{2} /(\mu \rho \lambda)}}, \quad \operatorname{Re} \mu_{1,2}<0$.

For convenience, the following notations are introduced:

$$
\sqrt{C+i D}=\mu_{1,2}=\frac{1}{\delta_{1,2}}+i k_{1,2}
$$

index 1 corresponds to the "-" sign, index 2 - to the "+" sign.

Wherein

$$
C=\frac{-\alpha A+B(\omega \mp 2 \Omega)}{A^{2}+B^{2}} ; \quad D=\frac{\alpha B+A(\omega \mp 2 \Omega)}{A^{2}+B^{2}},
$$

where, in turn, the $A$ and $B$ values are connected to the problem parameters by the following relations:

$$
A=v-\frac{\alpha B_{0}^{2}}{\mu \rho\left(\alpha^{2}+\omega^{2}\right)} ; B=-\frac{\omega B_{0}^{2}}{\mu \rho\left(\alpha^{2}+\omega^{2}\right)} .
$$


A.A. Gurchenkov

Then, the $\delta_{1,2}$ and $k_{1,2}$ values having physical meaning of the boundary layer thickness and of the wave number are determined by the following formulas:

$$
\frac{1}{\delta_{1,2}^{2}}=\frac{\sqrt{C^{2}+D^{2}}+C}{2} ; \quad k_{1,2}^{2}=\left(\frac{2}{\sqrt{C^{2}+D^{2}}-C}\right)^{-1} .
$$

Given these notations, the velocity field takes the following form:

$$
\begin{gathered}
\vec{v}(y, t)=e^{-\alpha t} e^{i \omega t}\left[\frac{\vec{u}(0)}{2}\left(e^{-\frac{y}{\delta_{1}}} e^{-i k_{1} y}+e^{-\frac{y}{\delta_{2}}} e^{i k_{2} y}\right)+\right. \\
\left.+i \frac{\vec{u}(0) \times \vec{e}_{y}}{2}\left(e^{-\frac{y}{\delta_{1}}} e^{-i k_{1} y}-e^{-\frac{y}{\delta_{2}}} e^{i k_{2} y}\right)\right]= \\
=\frac{\vec{u}(0)+i \vec{u}(0) \times \vec{e}_{y}}{2} e^{-\alpha t} e^{-\frac{y}{\delta_{1}}} e^{-i\left(\omega t+k_{1} y\right)}+ \\
+\frac{\vec{u}(0)-i \vec{u}(0) \times \vec{e}_{y}}{2} e^{-\alpha t} e^{-\frac{y}{\delta_{2}}} e^{i\left(\omega t+k_{2} y\right)} .
\end{gathered}
$$

Finally, we have

$$
\vec{v}=\hat{\mathrm{A}}_{1} e^{-i\left(k_{1} y-\omega t\right)}+\hat{\mathrm{A}}_{2} e^{i\left(k_{2} y+\omega t\right)} .
$$

Where

$$
\hat{\mathrm{A}}_{1}=\frac{1}{2}\left[\vec{u}(0)+i \vec{u}(0) \times \vec{e}_{y}\right] e^{-\alpha t} e^{-\frac{y}{\delta_{1}}}, \hat{\mathrm{A}}_{2}=\frac{1}{2}\left[\vec{u}(0)-i \vec{u}(0) \times \vec{e}_{y}\right] e^{-\alpha t} e^{-\frac{y}{\delta_{2}}} .
$$

Velocity field of the electrically conductive liquid is presented in the form of two plane waves induced by the plate damped harmonic oscillations. Phase velocities of these waves are, as follows:

$$
\begin{aligned}
& v_{1 \Phi}=\frac{\omega}{k_{1}}=\frac{\omega \sqrt{2}}{\left(\sqrt{\frac{\alpha^{2} A^{2}+B^{2}(\omega-2 \Omega)^{2}}{A^{2}+B^{2}}}-\frac{B(\omega-2 \Omega)-\alpha A}{A^{2}+B^{2}}\right)^{\frac{1}{2}}} ; \\
& v_{2 \Phi}=\frac{\omega}{k_{2}}=\frac{\omega \sqrt{2}}{\left(\sqrt{\frac{\alpha^{2} A^{2}+B^{2}(\omega+2 \Omega)^{2}}{A^{2}+B^{2}}}-\frac{B(\omega+2 \Omega)-\alpha A}{A^{2}+B^{2}}\right)^{\frac{1}{2}}} .
\end{aligned}
$$


In this case, the wave numbers have the following forms:

$$
\begin{aligned}
& k_{1}=\frac{1}{\sqrt{2}}\left(\sqrt{\frac{\alpha^{2}+(\omega-2 \Omega)^{2}}{A^{2}+B^{2}}}-\frac{B(\omega-2 \Omega)-\alpha A}{A^{2}+B^{2}}\right)^{\frac{1}{2}} ; \\
& \delta_{1}=\frac{\sqrt{2}}{\left(\sqrt{\frac{\alpha^{2}+(\omega-2 \Omega)^{2}}{A^{2}+B^{2}}}+\frac{B(\omega-2 \Omega)-\alpha A}{A^{2}+B^{2}}\right)^{\frac{1}{2}}},
\end{aligned}
$$

where $A^{2}+B^{2}=v^{2}+\frac{B_{0}^{2}\left(B_{0}^{2}-2 v \alpha \mu \rho\right)}{\mu^{2} \rho^{2}\left(\alpha^{2}+\omega^{2}\right)}$.

Let us consider the resonance case $\omega=2 \Omega$, then

$$
\begin{aligned}
& k_{1}=\frac{1}{\sqrt{2}\left(\sqrt{\frac{\alpha^{2}}{A^{2}+B^{2}}}+\frac{\alpha A}{A^{2}+B^{2}}\right)^{\frac{1}{2}}} ; \\
& \delta_{1}=\frac{\sqrt{2}}{\left(\sqrt{\frac{\alpha^{2}}{A^{2}+B^{2}}}-\frac{\alpha A}{A^{2}+B^{2}}\right)^{\frac{1}{2}}} .
\end{aligned}
$$

Let us choose the $B_{0}^{2}=2 v \alpha \mu \rho$ field induction. Then $A^{2}+B^{2}=v^{2}$;

$$
A=v-\frac{\alpha \cdot 2 v \alpha \mu \rho}{\mu^{2} \rho^{2}\left(\alpha^{2}+4 \Omega^{2}\right)}=v-\frac{v \cdot 2 \alpha^{2}}{\alpha^{2}+4 \Omega^{2}}=v \frac{4 \Omega^{2}-\alpha^{2}}{\alpha^{2}+4 \Omega^{2}} .
$$

In this case

$$
k_{1}=\sqrt{\frac{\alpha}{v}} \frac{1}{\sqrt{1+\alpha^{2} /\left(4 \Omega^{2}\right)}} ; \delta_{1}=\sqrt{\frac{\nu}{\alpha}} \sqrt{1+\frac{4 \Omega^{2}}{\alpha^{2}}} .
$$

The $k_{1}$ wave number and the $\delta_{1}$ boundary layer value do not dependent on the fluid magnetic permeability and electrical conductivity, and are determined only by the $\alpha$ attenuation coefficient and the $v$ fluid viscosity.

Nature of the second wave propagation does not depend on the features of the first plane wave motion.

Fig. 2 and 3 present dependences of the boundary layer and of the wave number on the plate oscillation frequency. The relations found completely solve the problem. 


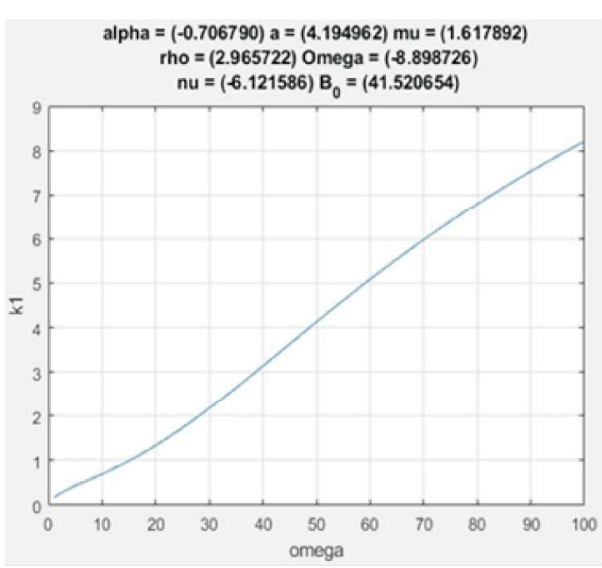

$a$

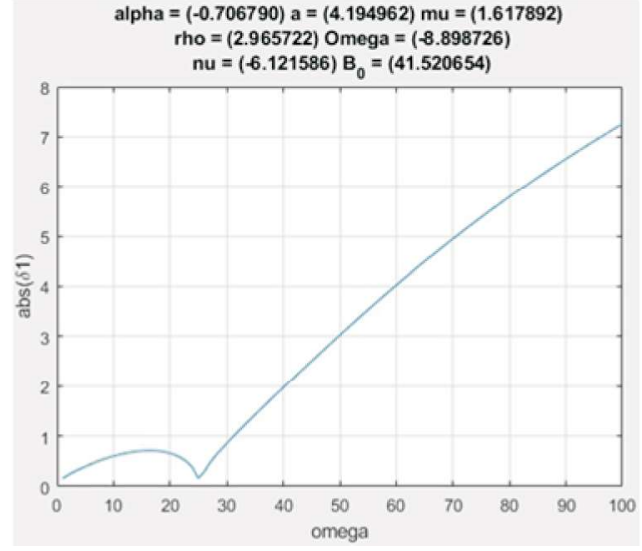

$b$

Fig. 2. $k_{1}$ wave number $(a)$ and $\delta_{1}$ boundary layer $(b)$

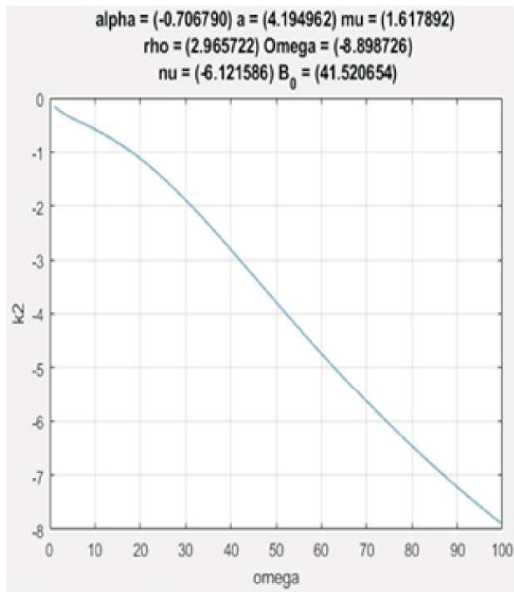

$a$

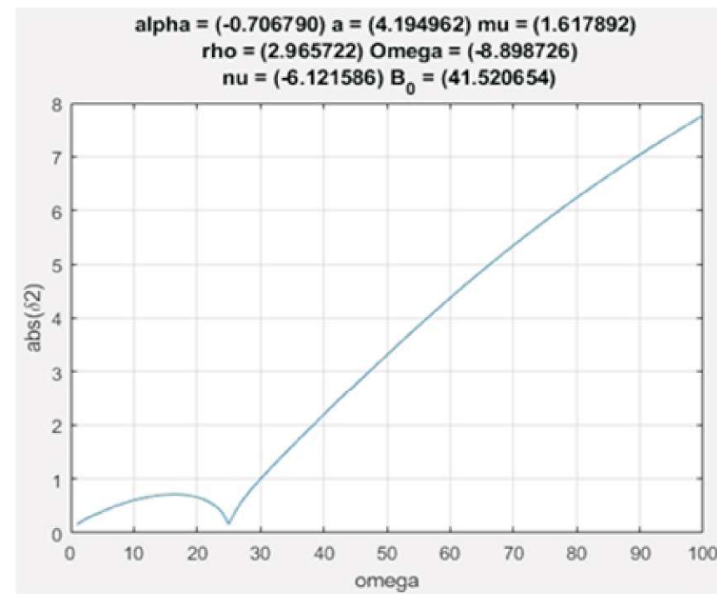

$b$

Fig. 3. $k_{2}$ wave number $(a)$ and $\delta_{2}$ boundary layer $(b)$

Conclusion. Analysis of the problem was carried out in regard to unsteady flow of a viscous electrically conductive incompressible fluid in a plane-parallel configuration. Exact solutions of three-dimensional nonstationary equations of magnetic hydrodynamics were found. In this case, no restrictions on the nature of the plate motion are imposed. Velocity field in the flow and shear stress vectors acting from the fluid upon the wall were determined. In regard to the problem of normal wall oscillations, the resonance case was considered and the structure of boundary layers adjacent to the plate was investigated. Mathematical procedure for integrating the system of differential equations within the problem examined could be used to study more complicated problems. In addition, the 
Unsteady Motion of Viscous Electrically Conductive Fluid Rotating in Half-Space Bounded...

results obtained could be used to account for the force effects during fluid motion in channels of various shapes, as well as in filtering problems and in simulating various physical phenomena in the moving fluid.

Translated by K. Zykova

\section{REFERENCES}

[1] Slezkin N.A. Dinamika vyazkoy neszhimaemoy zhidkosti [Dynamics of viscous incompressible fluid]. Moscow, Gostekhizdat Publ., 1955.

[2] Thornley Cl. On Stokes and Rayleigh layers in a rotating system. Q. J. Mech. Appl. Math., 1968, vol. 21, no. 4, pp. 451-461. DOI: 10.1093/qjmam/21.4.451

[3] Gurchenkov A.A., Yalamov Yu.I. Non-steady-state flow at a porous plate with blowing (suction) of the medium. J. Appl. Mech. Tech. Phys., 1980, vol. 21, no. 4, pp. 488-491.

DOI: $10.1007 / \mathrm{BF} 00916482$

[4] Landau L.D., Lifshits E.M. Teoreticheskaya fizika. T. 8. Elektrodinamika sploshnykh sred [Theoretical physics. Vol. 8. Electrodynamics of continuous media]. Moscow, Fizmatlit Publ., 2005.

[5] Alfwen H., Falthammar C.G. Cosmical electrodynamics. Fundamental principles. Claredon Press, 1963.

[6] Gurchenkov A.A. Dinamika zavikhrennoy zhidkosti v polosti vrashchayushchegosya tela [Dynamics of swirling fluid in a cavity of rotating body]. Moscow, Fizmatlit Publ., 2010.

[7] Gurchenkov A.A. The unsteady motion of a viscous fluid between rotating parallel walls. J. Appl. Math. Mech., 2002, vol. 66, no. 2, pp. 239-243.

DOI: $10.1016 /$ S0021-8928(02)00029-1

[8] Sivukhin D.V. Obshchiy kurs fiziki. T. 3. Elektrichestvo [General course of physics. Vol. 3. Electricity]. Moscow, Fizmatlit Publ., 2009.

[9] Xu C., Schuster E., Vazquez R., et al. Stabilization of linearized 2D magnetohydrodynamic channel flow by backstepping boundary control. Syst. Control Lett., 2008, vol. 57, no. 10, pp. 805-812. DOI: 10.1016/j.sysconle.2008.03.008

[10] Xu C., Schuster E., Vazquez R., et al. MHD channel flow control in 2D: Mixing enhancement by boundary feedback. Automatica, 2008, vol. 44, no. 10, pp. 2498-2507.

DOI: 10.1016/j.automatica.2008.02.018

[11] Grigoriadisa D.G.E., Kassinos S.C., Votyakova E.V. Immersed boundary method for the MHD flows of liquid metals. J. Comput. Phys., 2009, vol. 228, no. 3, pp. 903-920.

DOI: $10.1016 /$ j.jcp.2008.10.017

[12] Chebotarev A.Yu. Control of magnetohydrodynamic flow in the formation of a magnetic field with a prescribed configuration. Comput. Math. Math. Phys., 2009, vol. 49, no. 11, pp. 1913-1920. DOI: 10.1134/S0965542509110086

[13] Stepanov R.A., Chupin A.V., Frik P.G. Screw dynamo in a torus. Vychislitel'naya mekhanika sploshnykh sred [Computational Continuum Mechanics], 2008, vol. 1, no. 1, pp. 109-117 (in Russ.).

ISSN 0236-3941. Вестник МГТУ им. Н.Э. Баумана. Сер. Машиностроение. 2020. № 1 
A.A. Gurchenkov

[14] Noskov V., Denisov S., Frick P., et al. Magnetic field rotation in the screw gallium flow. Eur. Phys. J. B, 2004, vol. 41, no. 4, pp. 561-568. DOI: 10.1140/epjb/e2004-00349-8

[15] Rogachevskii I., Kleeorin N. Nonlinear theory of a "shear-current" effect and meanfield magnetic dynamos. Phys. Rev. E, 2004, vol. 70, no. 4, art. 046310.

DOI: 10.1103/PhysRevE.70.046310

[16] Alexakis A., Mininni P.D., Pouquet A. Shell-to-shell energy transfer in magnetohydrodynamics. I. Steady state turbulence. Phys. Rev. E, 2005, vol. 72, no. 4, art. 46301.

DOI: 10.1103/PhysRevE.72.046301

[17] Plunian F., Stepanov R. A non-local shell model of hydrodynamic and magnetohydrodynamic turbulence. New J. Phys., 2007, vol. 9, art. 294.

DOI: $10.1088 / 1367-2630 / 9 / 8 / 294$

[18] Radier K.-H., Stepanov R. Mean electromotive force due to turbulence of a conducting fluid in the presence of mean flow. Phys. Rev. E, 2006, vol. 73, no. 5, art. 056311.

DOI: 10.1103/PhysRevE.73.056311

[19] Denisov S.A., Noskov V.I., Stepanov R.A., et al. Measurements of turbulent magnetic diffusivity in a liquid-gallium flow. JETP Lett., 2008, vol. 88, no. 3, pp. 167-171.

DOI: $10.1134 /$ S0021364008150058

Gurchenkov A.A. - Dr. Sc. (Phys.-Math.), Professor, Department of Higher Mathematics, Bauman Moscow State Technical University (2-ya Baumanskaya ul. 5, str. 1, Moscow, 105005 Russian Federation); Leading Research Fellow, Federal Research Centre for Computer Science and Control, Russian Academy of Sciences (Vavilova ul. 40, Moscow, 119333 Russian Federation).

\section{Please cite this article as:}

Gurchenkov A.A. Unsteady motion of viscous electrically conductive fluid rotating in half-space bounded by a wall in the presence of medium injection (suction). Herald of the Bauman Moscow State Technical University, Series Mechanical Engineering, 2020, no. 1, pp. 107-118. DOI: https://doi.org/10.18698/0236-3941-2020-1-107-118 